\title{
Intermolecular Ene Reactions utilizing Oxazolones and Enol Ethers
}

Jason S. Fisk and Jetze J. Tepe

\section{Supporting Information:}

\section{General Methods:}

Reactions were carried out in flame-dried glassware under nitrogen atmosphere. Commercial solvents and reagents were used as received. Anhydrous methylene chloride was dispensed from a delivery system which passes the solvents through a column packed with dry neutral alumina. All reactions were magnetically stirred and monitored by TLC with $0.25 \mu \mathrm{m}$ pre-coated silica gel plates. Column chromatography was carried out on Silica Gel 60 (230-400 mesh) supplied by EM Science. Yields refer to chromatographically and spectroscopically pure compounds unless otherwise noted. Infrared spectra were recorded on a Nicolet IR/42 spectrometer. $1 \mathrm{H}$ and 13C NMR spectra were recorded on a Varian Inova-300 spectrometer, a Varian Gemini-300 spectrometer and a Varian Unity Plus-500 spectrometer. Chemical shifts are reported relative to the residue peaks of the solvent $\mathrm{CDCl}_{3}(7.24 \mathrm{ppm}$ for $1 \mathrm{H}$ and $77.0 \mathrm{ppm}$ for 13C). HRMS were obtained at the Mass Spectrometry Facility of Michigan State University with a JEOL JMS HX-110 mass spectrometer. Gas chromatography / low resolution mass spectra were recorded on a Hewlet-Packard 5890 Series II gas chromatograph connected to a TRIO-1 EI mass spectrometer.

\section{Procedures for Synthesis of Oxazolones:}

1. 2-Phenyl-4-carbmethoxy-5-oxazolone and 2-phenyl-4- $\alpha$-hydroxyethylidene-5oxazol- one were prepared in the same manner as had been previously reported. ${ }^{1}$

2. All other oxazolones were prepared as follows: A solution consisting of N-benzoyl amino acid $(1 \mathrm{mmol})$ and trifluoroacetic anhydride $(1 \mathrm{mmol})$ in dry dichloromethane 
$(50 \mathrm{~mL})$ was placed under nitrogen atmosphere and stirred at room temperature overnight. The reaction mixture was washed exhaustively with saturated $\mathrm{NaHCO}_{3}$ solution and brine $(25 \mathrm{~mL}$ each). Lastly, the solution was dried over anhydrous magnesium sulfate and the solvent was evaporated to dryness in vacuo. The resulting oxazolones were isolated as either solids or oils and were used without further purification. $^{2}$

\section{Procedures for Synthesis of Enol Ethers:}

The enol ethers used in entries 1-6 and 9-14 were purchased and used as received. All other enol ethers used in this study were prepared using known literature procedures. ${ }^{3-6}$

\section{General Procedure for Conia-Ene Reaction:}

A solution of oxazolone $(0.5 \mathrm{mmol})$ and enol ether $(0.6 \mathrm{mmol}$ to $1.5 \mathrm{mmol})$ in dry dichloromethane was stirred under nitrogen atmosphere in a flame dried flask for the requisite amount of time as monitored by TLC. The solvent was removed under vacuum and replaced with $\mathrm{MeOH}$. Completion of oxazolone ring opening was monitored by TLC. The solvent was removed under vacuum and the product was isolated if necessary by column chromatography on silica gel with an ethyl acetate / hexanes mixture.

Dimethyl-2-(1-tert-butoxyethyl)-2-(benzamido)malonate (1): Using the general $\mathrm{MeO}_{2} \mathrm{C} \mathrm{O}_{\mathrm{O}} \mathrm{CO}_{2} \mathrm{Me}$
$\mathrm{BzHN}$ $\begin{aligned} & \text { procedure, a solution of 2-phenyl-4-carbmethoxy-5(4H)-oxazolone } \\ & \mathrm{mL} \text { of anhydrous } \mathrm{CH}_{2} \mathrm{Cl}_{2} \text { was stirred at room temperature for } 30 \mathrm{~min} \text {. }\end{aligned}$ The resulting ene adduct was stirred in $20 \mathrm{~mL} \mathrm{MeOH}$ overnight, after which $0.16 \mathrm{~g}$ of malonate 1 was obtained ( $99 \%$ yield) as a clear oil. ${ }^{1} \mathrm{H}$ NMR (300 MHz), $\mathrm{CDCl}_{3}: \delta 1.10$ (s, 9H), $1.36(\mathrm{~d}, \mathrm{~J}=6 \mathrm{~Hz}, 3 \mathrm{H}), 3.73$ (s, 3H), 3.77 (s, 3H), 4.58 (q, J = 6, 1H), 7.23 (bs, $1 \mathrm{H}), 7.38-7.58(\mathrm{~m}, 3 \mathrm{H}), 7.78-7.82(\mathrm{~m}, 2 \mathrm{H}) ;{ }^{13} \mathrm{C} \mathrm{NMR}+$ DEPT $(75 \mathrm{MHz}) \mathrm{CDCl}_{3}: 19.23$ (-CH3), 28.84 (-CH3), 52.74 (-CO2 CH3), 53.20 (-CO2 CH3), 70.59 (quaternary C), $71.52(-\mathbf{C H}), 74.43$ (quaternary C), 127.20 (aromatic $\mathbf{C H}$ ), 128.56 (aromatic $\mathbf{C H}$ ), 131.79 (aromatic CH), 133.75 (aromatic quaternary C), 166.41, 166.62, 168.14; IR $\left(\mathrm{cm}^{-1}\right)$ : 3430, 
2979, 1748, 1676; HRMS (FAB): $m / z$ calcd for $\mathrm{C}_{18} \mathrm{H}_{26} \mathrm{NO}_{6}[\mathrm{M}+\mathrm{H}], 352.1757$; found, 352.1760 .

Dimethyl-2-(benzamido)-2-(1-butoxyethyl)malonate (2): Using the general procedure, $\mathrm{MeO}_{2} \mathrm{C} \underbrace{\mathrm{CO}_{2} \mathrm{Me}}_{\mathrm{O}_{\mathrm{Bu}}}$

a solution of 2-phenyl-4-carbmethoxy-5(4H)-oxazolone $(0.1 \mathrm{~g}, 0.46$ $\mathrm{mmol})$ and n-butyl vinyl ether $(0.06 \mathrm{~g}, 0.59 \mathrm{mmol})$ in $20 \mathrm{~mL}$ of anhydrous $\mathrm{CH}_{2} \mathrm{Cl}_{2}$ was stirred at room temperature for $30 \mathrm{~min}$. The resulting ene adduct was stirred in $20 \mathrm{~mL} \mathrm{MeOH}$ overnight, after which $0.16 \mathrm{~g}$ of malonate 2 was obtained (98\% yield) as a clear oil. ${ }^{1} \mathrm{H}$ NMR (300 MHz), $\mathrm{CDCl}_{3}: \delta 0.85$ (t, J $=7 \mathrm{~Hz}, 3 \mathrm{H}), 1.22-1.34(\mathrm{~m}, 2 \mathrm{H}), 1.32(\mathrm{~d}, \mathrm{~J}=6 \mathrm{~Hz}, 3 \mathrm{H}), 1.37-1.48(\mathrm{~m}, 2 \mathrm{H}), 3.16-3.24$ (m, 1H), 3.51-3.59 (m, 1H), 3.73 (s, 3H), 3.81 (s, 3H), 4.33 (q, J = 6 Hz, 1H), 7.32 (bs, 1H), 7.40-7.54 (m, 3H), 7.80-7.83 (m, 2H); ${ }^{13} \mathrm{C} \mathrm{NMR} \mathrm{+} \mathrm{DEPT} \mathrm{(75} \mathrm{MHz)} \mathrm{CDCl}_{3}: 13.76$ (-CH3), 14.78 (-CH3), 19.21 (-CH2), 31.84 (-CH2), 52.83 (-CO2CH3), 53.40 ($\mathrm{CO} 2 \mathrm{CH} 3$ ), $68.95(-\mathbf{C H} 2), 69.90$ (quaternary $\mathrm{C}), 78.09(-\mathbf{C H}), 127.20$ (aromatic $\mathbf{C H}$ ), 128.55 (aromatic $\mathbf{C H}$ ), 131.87 (aromatic $\mathbf{C H}$ ), 133.44 (aromatic quaternary C), 166.33, 166.44, 168.08; IR $\left(\mathrm{cm}^{-1}\right): 3424,2957,1747,1676$; HRMS (FAB): $\mathrm{m} / \mathrm{z}$ calcd for $\mathrm{C}_{18} \mathrm{H}_{26} \mathrm{NO}_{6}[\mathrm{M}+\mathrm{H}], 352.1759$; found, 352.1760 .

\section{Methyl-4,5-dihydro-4-(2-methoxypropan-2-yl)-5-oxo-2-phenyloxazole-4-carboxylate}

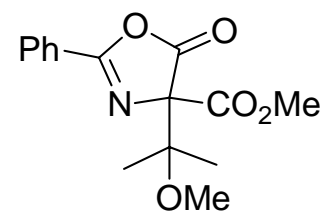

(3): A solution of 2-phenyl-4-carbmethoxy-5(4H)-oxazolone $(0.2 \mathrm{~g}$, $0.91 \mathrm{mmol})$ and 2-methoxy propene $(0.09 \mathrm{~g}, 1.19 \mathrm{mmol})$ in $20 \mathrm{~mL}$ of anhydrous $\mathrm{CH}_{2} \mathrm{Cl}_{2}$ was stirred at room temperature for $30 \mathrm{~min}$. The resulting ene adduct (3) was characterized by NMR. All attempts at ring opening the oxazolone with methanol were unsuccessful due to the reversibility of this reaction. According to the NMR spectra the initial ene reaction resulted in a yield of greater than 95\%. ${ }^{1} \mathrm{H}$ NMR $(300 \mathrm{MHz}), \mathrm{CDCl}_{3}: \delta 1.52(\mathrm{~s}, 3 \mathrm{H}), 1.55$ $(\mathrm{s}, 3 \mathrm{H}), 3.12(\mathrm{~s}, 3 \mathrm{H}), 3.76(\mathrm{~s}, 3 \mathrm{H}), 7.40(\mathrm{~m}, 3 \mathrm{H}), 7.99(\mathrm{~m}, 2 \mathrm{H}) ;{ }^{13} \mathrm{C}$ NMR $(75 \mathrm{MHz})$ $\mathrm{CDCl}_{3}: 18.66,20.39,49.59,53.22,79.81,82.57,125.49,128.30,128.68,133.00,163.13$, 165.01, 172.57; IR ( $\left.\mathrm{cm}^{-1}\right): 2953,1825,1755,1651,1066$. 
Dimethyl-2-(benzamido)-2-(tetrahydrofuran-2-yl)malonate (4): Using the general $\mathrm{MeO}_{2} \mathrm{C}$ procedure, a solution of 2-phenyl-4-carbmethoxy-5(4H)-oxazolone $(0.1 \mathrm{~g}, 0.46 \mathrm{mmol})$ and 2,3-dihydrofuran $(0.04 \mathrm{~g}, 0.59 \mathrm{mmol})$ in $20 \mathrm{~mL}$ of anhydrous $\mathrm{CH}_{2} \mathrm{Cl}_{2}$ was stirred at room temperature for $2 \mathrm{hr}$. The resulting ene adduct was stirred in $20 \mathrm{~mL} \mathrm{MeOH}$ overnight, after which $0.14 \mathrm{~g}$ of malonate 4 was obtained (99\% yield) as a clear oil. ${ }^{1} \mathrm{H}$ NMR (300 MHz), $\mathrm{CDCl}_{3}: \delta$ 1.70-1.91 (m, 2H), 2.07-2.22 (m, 1H), 2.19-2.32 (m, 1H), 3.67-3.82 (m, 2H), $3.76(\mathrm{~s}$, 3H), 3.84 (s, 3H), 4.72 (t, J = 7 Hz, 1H), 7.38 (bs, 1H), 7.38-7.54 (m, 3H), 7.80-7.84 (m, $2 \mathrm{H}) ;{ }^{13} \mathrm{C} \mathrm{NMR}+$ DEPT $(75 \mathrm{MHz}) \mathrm{CDCl}_{3}: 26.01$ (-CH2), 27.40 (-CH2), 52.94 ($\mathrm{CO} 2 \mathrm{CH} 3), 53.77$ (-CO2CH3), 68.54 (quaternary $\mathrm{C}), 68.86(-\mathrm{CH} 2), 81.47(-\mathrm{CH}), 127.18$ (aromatic $\mathbf{C H}$ ), 128.63 (aromatic $\mathbf{C H}$ ), 132.02 (aromatic $\mathbf{C H}$ ), 133.27 (aromatic quaternary C), 166.43, 166.53, 168.08; IR $\left(\mathrm{cm}^{-1}\right): 3414,2955,1743,1670 ;$ HRMS (FAB): $m / z$ calcd for $\mathrm{C}_{16} \mathrm{H}_{20} \mathrm{NO}_{6}[\mathrm{M}+\mathrm{H}], 322.1290$; found, 322.1290 .

Dimethyl-2-(benzamido)-2-(tetrahydropyran-2-yl)malonate (5): Using the general $\mathrm{MeO}_{2} \mathrm{C}$ BzHN procedure, a solution of 2-phenyl-4-carbmethoxy-5(4H)-oxazolone $(0.1 \mathrm{~g}, 0.46 \mathrm{mmol})$ and 3,4-dihydro-2H-pyran $(0.05 \mathrm{~g}, 0.59 \mathrm{mmol})$ in $20 \mathrm{~mL}$ of anhydrous $\mathrm{CH}_{2} \mathrm{Cl}_{2}$ was stirred at room temperature for 22 $\mathrm{hr}$. The resulting ene adduct was stirred in $20 \mathrm{~mL} \mathrm{MeOH}$ overnight yielding $0.14 \mathrm{~g}$ of malonate 5 (99\% yield) as a clear colorless oil after silica gel chromatography (20\% ethyl acetate / 80\% hexanes). ${ }^{1} \mathrm{H}$ NMR (300 MHz), $\mathrm{CDCl}_{3}: \delta 1.26-1.64(\mathrm{~m}, 4 \mathrm{H}), 1.80$ $1.86(\mathrm{~m}, 1 \mathrm{H}), 2.10-2.18(\mathrm{~m}, 1 \mathrm{H}), 3.38-3.48(\mathrm{~m}, 1 \mathrm{H}), 3.73(\mathrm{~s}, 3 \mathrm{H}), 3.82(\mathrm{~s}, 3 \mathrm{H}), 3.90-3.96$ (m, 1H), $4.24(\mathrm{dd}, \mathrm{J}=12 \mathrm{~Hz}, \mathrm{~J}=2 \mathrm{~Hz}, 1 \mathrm{H}), 7.39$ (bs, 1H), 7.40-7.54 (m, 3H), 7.82-7.86 $(\mathrm{m}, 2 \mathrm{H}) ;{ }^{13} \mathrm{C} \mathrm{NMR}+$ DEPT $(75 \mathrm{MHz}) \mathrm{CDCl}_{3}: 22.97$ (-CH2), 25.71 (-CH2), 26.78 ($\mathbf{C H} 2), 52.82$ (-CO2CH3), 53.69 (-CO2CH3), 69.14 (quaternary C), 69.42 (-CH2), 80.99 (-CH), 127.22 (aromatic $\mathbf{C H}$ ), 128.54 (aromatic $\mathbf{C H}$ ), 131.90 (aromatic $\mathbf{C H}$ ), 133.33 (aromatic quaternary C), 165.99, 166.41, 168.16; IR $\left(\mathrm{cm}^{-1}\right)$ : 3422, 2953, 1745, 1674; HRMS (FAB): $\mathrm{m} / \mathrm{z}$ calcd for $\mathrm{C}_{17} \mathrm{H}_{22} \mathrm{NO}_{6}[\mathrm{M}+\mathrm{H}], 336.1445$; found, 336.1447 . 
Dimethyl-2-(benzamido)-2-(1-benzyloxy-2-methylpropyl)malonate (6): Using the

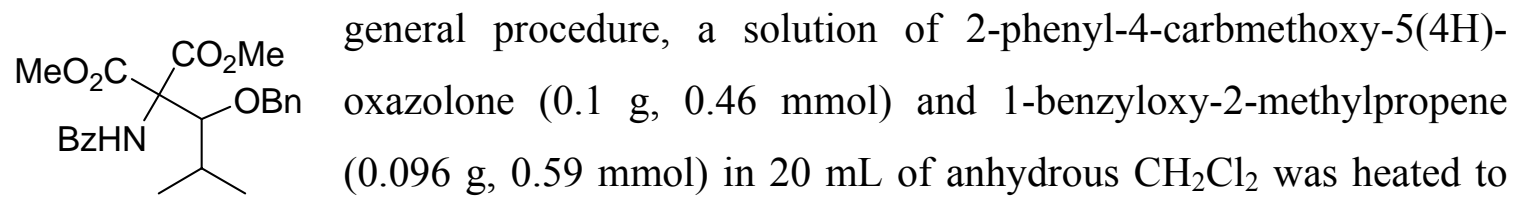
reflux for 48 hours. The resulting ene adduct was stirred in $20 \mathrm{~mL} \mathrm{MeOH}$ overnight yielding $0.13 \mathrm{~g}$ of malonate 6 (68\% yield) as a clear colorless oil after silica gel chromatography ( $20 \%$ ethyl acetate / $80 \%$ hexanes). ${ }^{1} \mathrm{H}$ NMR $(300 \mathrm{MHz}), \mathrm{CDCl}_{3}: \delta$ 0.92 (d, J = $7 \mathrm{~Hz}, 3 \mathrm{H}), 1.15$ (d, J = $7 \mathrm{~Hz}, 3 \mathrm{H}), 2.37-2.45$ (m, 1H), 3.70 (s, 3H), 3.77 (s, $3 \mathrm{H}), 4.44(\mathrm{~d}, \mathrm{~J}=2 \mathrm{~Hz}, 1 \mathrm{H}), 4.59(\mathrm{~d}, \mathrm{~J}=11 \mathrm{~Hz}, 1 \mathrm{H}), 4.68(\mathrm{~d}, \mathrm{~J}=11 \mathrm{~Hz}, 1 \mathrm{H}), 7.24-7.51$ (m, 9H), 7.77-7.80 (m, 2H); ${ }^{13} \mathrm{C} \mathrm{NMR} \mathrm{+} \mathrm{DEPT}(75 \mathrm{MHz}) \mathrm{CDCl}_{3}: 16.23(-\mathrm{CH} 3), 23.87$ (-CH3), $29.78(-\mathrm{CH}), 53.08$ (-CO2CH3), 53.39 (-CO2CH3), 70.09 (quaternary C), 75.31 (-CH2), $84.97(-\mathrm{CH}), 127.14$ (aromatic $\mathrm{CH}$ ), 127.42 (aromatic $\mathrm{CH}$ ), 127.51 (aromatic $\mathrm{CH}), 128.25$ (aromatic $\mathrm{CH}$ ), 128.49 (aromatic $\mathrm{CH}$ ), 128.63 (aromatic $\mathrm{CH}$ ), 131.90 (aromatic $\mathrm{CH}$ ), 133.44 (aromatic quaternary C), 138.26 (aromatic quaternary C), 166.41, 167.22, 168.35; IR $\left(\mathrm{cm}^{-1}\right): 3418,2955,1743,1672 ;$ HRMS (FAB): $\mathrm{m} / \mathrm{z}$ calcd for $\mathrm{C}_{23} \mathrm{H}_{28} \mathrm{NO}_{6}[\mathrm{M}+\mathrm{H}], 414.1919$; found, 414.1916.

Dimethyl-2-(benzamido)-2-(tetrahydro-3-methylfuran-2-yl)malonate (7): Using the

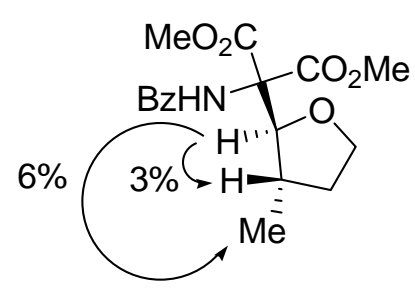
general procedure, a solution of 2-phenyl-4-carbmethoxy5(4H)-oxazolone (0.05 g, $0.23 \mathrm{mmol})$ and 2,3-dihydro-4methylfuran $(0.058 \mathrm{~g}, 0.68 \mathrm{mmol})$ in $20 \mathrm{~mL}$ of anhydrous $\mathrm{CH}_{2} \mathrm{Cl}_{2}$ was heated to reflux for 24 hours. The resulting ene adduct was stirred in $20 \mathrm{~mL} \mathrm{MeOH}$ overnight yielding $0.06 \mathrm{~g}$ of malonate 7 ( $81 \%$ yield) as a white solid after silica gel chromatography (40\% ethyl acetate / 60\% hexanes). ${ }^{1} \mathrm{H}$ NMR (300 MHz), $\mathrm{CDCl}_{3}: \delta 1.19(\mathrm{~d}, \mathrm{~J}=7 \mathrm{~Hz}, 3 \mathrm{H}), 1.48$ $1.56(\mathrm{~m}, 1 \mathrm{H}), 1.80-1.92(\mathrm{~m}, 1 \mathrm{H}), 2.70-2.80(\mathrm{~m}, 1 \mathrm{H}), 3.72-3.84(\mathrm{~m}, 1 \mathrm{H}), 3.77(\mathrm{~s}, 3 \mathrm{H})$, 3.82-3.88 (m, 1H), 3.84 (s, 3H), $3.41(\mathrm{~d}, \mathrm{~J}=5 \mathrm{~Hz}, 1 \mathrm{H}), 7.37$ (bs, 1H), 7.41-7.52 (m, 3H), 7.80-8.83 (m, 2H); ${ }^{13} \mathrm{C} \mathrm{NMR}+$ DEPT $(75 \mathrm{MHz}) \mathrm{CDCl}_{3}$ : 19.77 (-CH3), 34.65 (-CH2), $34.77(-\mathrm{CH}), 52.91$ (-CO2CH3), 53.83 (-CO2 CH3), 68.02 (-CH2), 68.94 (quaternary C), $88.13(-\mathrm{CH}), 127.18$ (aromatic $\mathrm{CH}), 128.68$ (aromatic $\mathrm{CH}), 132.06$ (aromatic $\mathrm{CH}$ ), 
133.28 (aromatic quaternary C), 166.50, 168.10; IR $\left(\mathrm{cm}^{-1}\right)$ : 3414, 2955, 1745, 1672; HRMS (FAB): $\mathrm{m} / \mathrm{z}$ calcd for $\mathrm{C}_{17} \mathrm{H}_{22} \mathrm{NO}_{6}[\mathrm{M}+\mathrm{H}], 336.1449$; found, 336.1447 .

4-acetyl-4-(1-tert-butoxyethyl)-2-phenyloxazol-5(4H)-one (10a): A solution of 2-<smiles>CC(=O)C(C)C1(C(C)C)N=C(c2ccccc2)OC1=O</smilesphenyl-4- $\alpha$-hydroxyethylidene-5-oxazolone $(0.1 \mathrm{~g}, 0.49 \mathrm{mmol})$ and tertbutyl vinyl ether $(0.06 \mathrm{~g}, 0.64 \mathrm{mmol})$ in $20 \mathrm{~mL}$ of anhydrous $\mathrm{CH}_{2} \mathrm{Cl}_{2}$ was stirred at room temperature for $24 \mathrm{hrs}$. The solution was washed twice with $20 \mathrm{~mL}$ of saturated $\mathrm{NaHCO}_{3}$ solution, once with brine and dried using magnesium sulfate. The solvent was removed under vacuum yielding $0.15 \mathrm{~g}$ of oxazolone 10a ( $1: 1.2)$ mixture of diastereomers) in a $98 \%$ yield as an orange oil. No further attempt was made to separate the two diastereomers. Diastereomer 1: ${ }^{1} \mathrm{H}$ NMR (300 MHz), $\mathrm{CDCl}_{3}: \delta 1.10$ (s, 9H), $1.24(\mathrm{~d}, 3 \mathrm{H}, \mathrm{J}=6 \mathrm{~Hz}), 2.36(\mathrm{~s}, 3 \mathrm{H}), 4.40(\mathrm{q}, 1 \mathrm{H}, \mathrm{J}=6$ $\mathrm{Hz}), 7.46-7.52(\mathrm{~m}, 2 \mathrm{H}), 7.55-7.62(\mathrm{~m}, 1 \mathrm{H}), 8.02-8.09(\mathrm{~m}, 2 \mathrm{H}) ;{ }^{13} \mathrm{C} \mathrm{NMR}+$ DEPT $(125$ MHz) $\mathrm{CDCl}_{3}: 17.49$ (-CH3), 28.46 (-CH3), 28.63 (-CH3), $71.41(-\mathrm{CH}), 75.34$ (quaternary C), 85.82 (quaternary C), 125.44 (aromatic quaternary C), 128.08 (aromatic $\mathrm{CH}$ ), 128.78 (aromatic CH), 132.99 (aromatic CH), 162.31, 171.72, 198.84; Diastereomer 2: ${ }^{1} \mathrm{H}$ NMR (300 MHz), $\mathrm{CDCl}_{3}: \delta 1.11(\mathrm{~s}, 9 \mathrm{H}), 1.29(\mathrm{~d}, 3 \mathrm{H}, \mathrm{J}=6 \mathrm{~Hz}), 2.25$ (s, 3H), 4.57 (q, 1H, J = $6 \mathrm{~Hz}), 7.46-7.52(\mathrm{~m}, 2 \mathrm{H}), 7.55-7.62(\mathrm{~m}, 1 \mathrm{H}), 8.02-8.09$ (m, 2H); ${ }^{13} \mathrm{C} \mathrm{NMR}$ + DEPT (125 MHz) $\mathrm{CDCl}_{3}: 18.79$ (-CH3), 28.49 (-CH3), 28.53 (-CH3), 71.70 $(-\mathrm{CH}), 74.94$ (quaternary $\mathrm{C}$ ), 87.47 (quaternary $\mathrm{C}$ ), 125.57 (aromatic quaternary C), 128.19 (aromatic CH), 128.70 (aromatic $\mathrm{CH}$ ), 132.94 (aromatic $\mathrm{CH}$ ), 162.65, 172.59, 199.32; IR $\left(\mathrm{cm}^{-1}\right): 2976,1836,1723,1653$.

Methyl-3-tert-butoxy-2-acetyl-2-(benzamido)butanoate (10b): The resulting ene

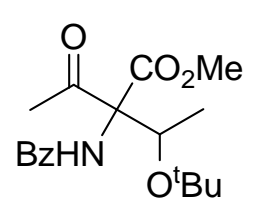
adduct was stirred in $20 \mathrm{~mL} \mathrm{MeOH}$ overnight, after which $0.07 \mathrm{~g}$ of ester 10 ( 1 : 1.2 mixture of diastereomers) was obtained ( $45 \%$ yield) as a clear oil after silica gel column chromatography ( $20 \%$ ethyl acetate / hexanes). No further attempt was made to separate the two diastereomers. ${ }^{1} \mathrm{H}$ NMR $(300 \mathrm{MHz})$, $\mathrm{CDCl}_{3}: \delta 1.11(\mathrm{~s}, 9 \mathrm{H}), 1.17(\mathrm{~s}, 9 \mathrm{H}), 1.26(\mathrm{~d}, \mathrm{~J}=6 \mathrm{~Hz}, 3 \mathrm{H}), 1.30(\mathrm{~d}, \mathrm{~J}=6 \mathrm{~Hz}, 3 \mathrm{H}), 2.12$ (s, $3 \mathrm{H}), 2.26(\mathrm{~s}, 3 \mathrm{H}), 3.75(\mathrm{~s}, 3 \mathrm{H}), 3.87(\mathrm{~s}, 3 \mathrm{H}), 4.65(\mathrm{q}, \mathrm{J}=6 \mathrm{~Hz}, 1 \mathrm{H}), 4.72(\mathrm{q}, \mathrm{J}=6 \mathrm{~Hz}$, 1H), 7.37 (bs, 1H), 7.42-7.54 (m, 3H), 7.81-7.87 (m, 2H); ${ }^{13} \mathrm{C}$ NMR + DEPT (75 MHz) 
$\mathrm{CDCl}_{3}$ : 18.99 (-CH3), 19.20 (-CH3), 26.06 (-CH3), 28.85 (-CH3), 29.01 (-CH3), 52.71 (-CO2CH3), 53.27 (-CO2CH3), 70.82 (-CH), 71.37 (-CH), 74.28 (quaternary C), 74.55 (quaternary C), 74.60 (quaternary $\mathrm{C}$ ), 76.35 (quaternary $\mathrm{C}$ ), 127.16 (aromatic $\mathrm{CH}$ ), 127.20 (aromatic CH), 128.59 (aromatic CH), 128.67 (aromatic $\mathbf{C H ) , ~} 131.83$ (aromatic $\mathbf{C H}$ ), 132.02 (aromatic $\mathbf{C H}$ ), 133.35 (aromatic quaternary C), 133.60 (aromatic quaternary C), 166.42, 166.53, 167.80, 168.51, 197.09, 198.92; IR ( $\left.\mathrm{cm}^{-1}\right): 3420,2978$, 1749, 1726, 1672; HRMS (FAB): $m / z$ calcd for $\mathrm{C}_{18} \mathrm{H}_{26} \mathrm{NO}_{5}[\mathrm{M}+\mathrm{H}], 336.1810$; found, 336.1811 .

Methyl-3-tert-butoxy-2-phenyl-2-(benzamido)butanoate (11): Using the general mmol $)$ and tert-butyl vinyl ether $(0.13 \mathrm{~g}, 1.26 \mathrm{mmol})$ in $20 \mathrm{~mL}$ of
anhydrous toluene was stirred at reflux for $8 \mathrm{hrs}$. The resulting ene adduct was reacted with $\mathrm{NaOMe}(0.42 \mathrm{mmol})$ in $20 \mathrm{~mL} \mathrm{MeOH}$ for one hour. The solvent was then removed under vacuum and the material was partitioned between $30 \mathrm{~mL}$ of $\mathrm{CH}_{2} \mathrm{Cl}_{2}$ and $30 \mathrm{~mL}$ of water. The organic layer was dried using magnesium sulfate and the solvent was removed under vacuum yielding $0.14 \mathrm{~g}$ of ester 11 (3: 1 mixture of diastereomers) in a 95\% yield as a yellow solid. No further attempt was made to separate the two diastereomers. Diastereomer 1: ${ }^{1} \mathrm{H} \mathrm{NMR}(500 \mathrm{MHz}), \mathrm{CDCl}_{3}: \delta 0.96(\mathrm{~s}, 9 \mathrm{H})$, 1.29 (d, 3H, J = 6 Hz), 3.75 (s, 3H), 4.36 (q, 1H, J = 6 Hz), 7.22-7.32 (m, 4H), 7.44-7.48 $(\mathrm{m}, 2 \mathrm{H}), 7.50-7.54(\mathrm{~m}, 1 \mathrm{H}), 7.58-7.62(\mathrm{~m}, 2 \mathrm{H}), 7.84-7.86(\mathrm{~m}, 2 \mathrm{H}) ; \quad{ }^{13} \mathrm{C} \mathrm{NMR}+$ DEPT (125 MHz) $\mathrm{CDCl}_{3}$ : 19.80 (-CH3), 28.43 (-CH3), 52.48 (-CO2CH3), 69.59 (quaternary C), 73.34 (-CH), 74.80 (quaternary C), 127.08 (aromatic $\mathbf{C H}$ ), 127.30 (aromatic $\mathbf{C H}$ ), 127.60 (aromatic $\mathbf{C H}$ ), 127.65 (aromatic $\mathbf{C H}$ ), 128.60 (aromatic $\mathbf{C H}$ ), 131.58 (aromatic $\mathrm{CH}$ ), 134.76 (aromatic quaternary C), 138.12 (aromatic quaternary C), 166.77, 171.12; Diastereomer 2: ${ }^{1} \mathrm{H}$ NMR (500 MHz), $\mathrm{CDCl}_{3}$ : 1.03 (d, 3H, J = $6 \mathrm{~Hz}$ ), 1.19 (s, 9H), 3.72 $(\mathrm{s}, 3 \mathrm{H}), 4.51(\mathrm{q}, 1 \mathrm{H}, \mathrm{J}=6), 7.28(\mathrm{~m}, 4 \mathrm{H}), 7.44-7.48(\mathrm{~m}, 2 \mathrm{H}), 7.50-7.54(\mathrm{~m}, 1 \mathrm{H}), 7.58-$ 7.62 (m, 2H), 7.84-7.86 (m, 2H); ${ }^{13} \mathrm{C}$ NMR + DEPT (125 MHz) $\mathrm{CDCl}_{3}$ : 19.06 (-CH3), 28.87 (-CH3), 52.66 (-CO2CH3), 70.59 (quaternary C), $72.66(-\mathrm{CH}), 74.83$ (quaternary C), 127.09 (aromatic CH), 127.27 (aromatic $\mathbf{C H}$ ), 127.41 (aromatic $\mathbf{C H}$ ), 127.83 (aromatic $\mathbf{C H}$ ), 128.60 (aromatic $\mathbf{C H}$ ), 131.50 (aromatic $\mathbf{C H}$ ), 135.14 (quaternary 
aromatic C), 136.21 (quaternary aromatic C), 167.55, 172.17; IR $\left(\mathrm{cm}^{-1}\right)$ : 3416, 2976, 1736, 1670; LRMS (EI) (m/z): 369.

4-(1-tert-butoxyethyl)-4-(naphthalen-1-yl)-2-phenyloxazol-5(4H)-one (12a): $\quad$ A solution of 2-phenyl-4-naphthyl-5(4H)-oxazolone $(0.18 \mathrm{~g}, 0.6 \mathrm{mmol})$
and tert-butyl vinyl ether $(0.19 \mathrm{~g}, 1.88 \mathrm{mmol})$ in $30 \mathrm{~mL}$ of anhydrous diastereomers) in a $99 \%$ yield as a yellow solid. No further attempt was made to separate the two diastereomers. ${ }^{1} \mathrm{H}$ NMR (500 MHz), $\mathrm{CDCl}_{3}: \delta 1.03$ (d, 3H, J = $\left.7 \mathrm{~Hz}\right), 1.12$, (d, $3 \mathrm{H}, \mathrm{J}=7 \mathrm{~Hz}$ ), 1.14 (s, 9H), 1.16 (s, 9H), 4.93 (q, 1H, J = $7 \mathrm{~Hz}$ ), 4.99 (q, 1H, J = $7 \mathrm{~Hz}$ ), 7.15-7.25 (m, 1H), 7.16-7.26 (m, 1H), 7.41-7.54 (m, 4H), 7.42-7.55 (m, 4H), 7.56-7.63 (m, 1H), 7.57-7.63 (m, 1H), $7.81(\mathrm{~d}, 1 \mathrm{H}, \mathrm{J}=8 \mathrm{~Hz}), 7.82(\mathrm{~d}, 1 \mathrm{H}, \mathrm{J}=8 \mathrm{~Hz}), 8.10(\mathrm{~d}, 2 \mathrm{H}, \mathrm{J}$ $=7 \mathrm{~Hz}), 7.92(\mathrm{~d}, 2 \mathrm{H}, \mathrm{J}=7 \mathrm{~Hz}), 8.05-8.07(\mathrm{~m}, 1 \mathrm{H}), 8.05-8.08(\mathrm{~m}, 1 \mathrm{H}), 8.10(\mathrm{~d}, 1 \mathrm{H}, \mathrm{J}=7$ $\mathrm{Hz}), 8.15$ (d, 1H, J = 7 Hz), 9.24 (d, 1H, J = 9 Hz), 9.29 (d, 1H, J = 9 Hz); ${ }^{13} \mathrm{C}$ NMR (125 MHz) $\mathrm{CDCl}_{3}: 17.18,18.47,28.71,71.63,72.91,74.70,74.99,81.38,81.96,124.57$, $124.95,125.00,125.26,125.43,125.64,125.76,125.79,126.27,126.34,126.71,127.07$, 127.21, 127.92, 128.14, 128.18, 128.65, 128.98, 129.07, 129.47, 129.76, 130.79, 131.41, $132.27,132.38,132.44,134.84,134.97,159.58,160.11,176.18,178.16 ; \quad I R\left(\mathrm{~cm}^{-1}\right)$ : 3150, 2976, 1810, 1692; LRMS (EI) (m/z): 388.

Methyl-3-tert-butoxy-2-(naphthalene-1-yl)-2-(benzamido)butanoate (12b): To flame<smiles>[R8]NC(c1cccc2ccccc12)(C(C)CCC)C(C)OC</smiles>
dried round bottom flask was added oxazolone 12a $(0.19 \mathrm{~g}, 0.5 \mathrm{mmol})$ in $20 \mathrm{~mL}$ of $\mathrm{MeOH}$. The solution was treated with $\mathrm{NaOMe}$ solution $(1 \mathrm{M}$ in $\mathrm{MeOH}, 2 \mathrm{mmol}$ ) for $36 \mathrm{hrs}$ at room temperature. The solvent was then removed under vacuum and the material was partitioned between $30 \mathrm{~mL}$ of $\mathrm{CH}_{2} \mathrm{Cl}_{2}$ and $30 \mathrm{~mL}$ of water. The organic layer was dried using magnesium sulfate and the solvent was removed under vacuum yielding $0.11 \mathrm{~g}$ of ester 12b (2: 1 mixture of diastereomers) in a 52\% yield as a yellow solid. No further attempt was made to separate the two diastereomers. ${ }^{1} \mathrm{H}$ NMR (500 MHz), $\mathrm{CDCl}_{3}: \delta 1.05$ (s, 
9H), $1.23(\mathrm{~s}, 9 \mathrm{H}), 1.55(\mathrm{~d}, 2 \mathrm{H}, \mathrm{J}=6 \mathrm{~Hz}), 1.62(\mathrm{~d}, 2 \mathrm{H}, \mathrm{J}=6 \mathrm{~Hz}), 3.57$ (s, 3H), 3.67 (s, $3 \mathrm{H}), 5.06(\mathrm{q}, 1 \mathrm{H}, \mathrm{J}=6 \mathrm{~Hz}), 5.31(\mathrm{q}, 1 \mathrm{H}, \mathrm{J}=6 \mathrm{~Hz}), 7.29-7.51(\mathrm{~m}, 12 \mathrm{H}), 7.57(\mathrm{~d}, 1 \mathrm{H}, \mathrm{J}=7$ $\mathrm{Hz}), 7.65(\mathrm{~d}, 1 \mathrm{H}, \mathrm{J}=6 \mathrm{~Hz}), 7.74-7.82(\mathrm{~m}, 8 \mathrm{H}), 7.85-7.93(\mathrm{~m}, 2 \mathrm{H}), 8.01(2,1 \mathrm{H}, \mathrm{J}=9 \mathrm{~Hz})$, $8.26(\mathrm{~d}, 1 \mathrm{H}, \mathrm{J}=9 \mathrm{~Hz}) ;{ }^{13} \mathrm{C} \mathrm{NMR}+$ DEPT (125 MHz) $\mathrm{CDCl}_{3}: 20.38$ (-CH3), 21.51 (CH3), 28.77 (-CH3), 29.15 (-CH3) 52.73 (-CO2CH3), 52.99 (-CO2CH3), 69.05, 69.35, $71.06,72.87,74.40,74.66,123.18,123.36,124.16,124.72,124.85,125.20,125.41$, $126.03,126.22$, 126.99, 127.03, 127.85, 128.44, 128.97, 129.29, 129.52, 131.16, 131.30, 131.46, 131.63, 132.59, 132.75, 133.90, 134.30, 135.11, 135.30, IR $\left(\mathrm{cm}^{-1}\right): 3414,2976$, 1736, 1672; LRMS (EI) (m/z): 420.

Dimethyl-2-(benzamido)-2-(3-deuterotetrahydropyran-2-yl)malonate (14): Using $\mathrm{MeO}_{2} \mathrm{C}-\mathrm{CO}_{2} \mathrm{Me}$ the general procedure, a solution of 2-phenyl-4-carbmethoxy-5(4H)oxazolone (0.02 g, $0.09 \mathrm{mmol})$ and a large excess of 5-deutero-3,4dihydropyran (50\% deuterium incorporated) in $20 \mathrm{~mL}$ of anhydrous $\mathrm{CH}_{2} \mathrm{Cl}_{2}$ was stirred at room temperature for $18 \mathrm{hr}$. The resulting ene adduct was stirred in $20 \mathrm{~mL} \mathrm{MeOH}$ overnight yielding $0.03 \mathrm{~g}$ of malonate 14 ( $99 \%$ yield) as a clear colorless oil after silica gel chromatography (25\% ethyl acetate / 75\% hexanes). ${ }^{1} \mathrm{H}$ NMR (300 MHz), $\mathrm{CDCl}_{3}$ : $\delta$ 1.29-1.59 (m, 4H), 1.81-1.84 (m, 1H), $2.13(\mathrm{~d}, \mathrm{~J}=15$, $0.5 \mathrm{H}), 3.43$ (td, J = $7 \mathrm{~Hz}, \mathrm{~J}=2 \mathrm{~Hz}, 1 \mathrm{H}), 3.73$ (s, 3H), 3.82 (s, 3H), 3.91-3.94 (m, 1H), 4.22-4.25 (m, 1H), 7.39 (bs, 1H), 7.42-7.45 (m, 2H), 7.50-7.52 (m, 1H), 7.83-7.85 (m, $2 \mathrm{H}) ;{ }^{13} \mathrm{C} \mathrm{NMR}+$ DEPT $(75 \mathrm{MHz}) \mathrm{CDCl}_{3}: 22.92$ (-CH2), 25.72 (-CH2), 26.82 (-CH2), 52.84 (-CO2CH3), 53.69 (-CO2CH3), 69.20 (quaternary C), 69.45 (-CH2), 80.99 (-CH), 127.26 (aromatic CH), 128.57 (aromatic $\mathbf{C H}$ ), 131.91 (aromatic $\mathbf{C H}), 133.41$ (aromatic quaternary C), 166.03, 166.44, 168.19; IR ( $\left.\mathrm{cm}^{-1}\right)$ : 3422, 2953, 1745, 1674;

\section{References:}

1. Cornforth, J. W.; Clarke, H. T.; et al. Oxazoles and oxazolones. Chemistry of Penicillin. (1949), 688-848.

2. Chen, F. M. F.; Kuroda, K.; Benoiton, N. L. Synthesis, 1979, 230-232.

3. Barbot, F.; Miginiac, P.; Helv. Chim. Acta. 1979, 1451-1457.

4. Bedoukian, P. Z.; J. Am. Chem. Soc. 1944, 66, 1325-1327. 
5. Temme, O.; Taj, S.; Andersson, P. G. J. Org. Chem. 1998, 63, 6007-6015.

6. Frimer, A.; Bartlett, P.; Boschung, A.; Jewett, J. J. Am. Chem. Soc. 1977, 99, 7977-7986. 